\section{How to use social media for fisheries science and management professionals.}

While conservation scientists often use social media to engage the public and collaborate on scientific endeavors, fisheries scientists and managers are less represented online. In this paper, the author explains some key benefits and methods for using social media to encourage others to join the conversation.

\section{Twitter}

One of the most popular social media platforms with scientists is Twitter. Many journalists are on the platform, allowing scientists to share information with the media - and to correct misinformation. Information is shared on Twitter via tweets, which may contain text, images, video, or links, so long as the contents do not exceed a 280 -character limit.

Users follow Twitter accounts to see that account's tweets. If a user follows you, they will see your tweets, but you will not automatically see theirs. Tweets may be retweeted to share that tweet with the user's followers, increasing the reach of an individual tweet beyond the followers of the original tweet's author.

Creating a Twitter account is free and quick. Scientists tend to choose a handle (username) that is either their name, a description of their work, or a combination of the two. For example, the author of this paper is @WhySharksMatter, while the author of this summary is @nwehner. Organizations tend to use their names/acronyms, as MarXiv does with @MarXivPapers.

Your biography on Twitter is useful to explain your expertise. One link may be included in your biography, which managers often use to link to their department's official website. The author recommends establishing separate institutional accounts from personal accounts, but not separating personal from professional Twitter content unless prohibited by your employer.

\section{Facebook}

Despite nearly 4/5 Americans using Facebook (versus nearly 1/4 for Twitter), Facebook is less useful for public outreach. Namely, because Facebook chooses which content to show a user in their newsfeed based on an algorithm. Facebook friends must be manually approved. Unlike Twitter, sharing is reciprocal among friends and there is no character limit.

Facebook's fan pages work more like Twitter, where a user may like a fan page of your organization without requiring your approval. Users who like your fan page will see your updates, but you will not automatically see theirs. You will need a free, personal Facebook account to create a fan page. Sharing content through your fan page is nearly identical as sharing content on your personal account.

Facebook groups (which also require a personal account) allow users to coalesce around similar interests without the need to friend one another. Groups allow for various privacy settings, giving the host control over who can join or post to a group. Sharing research and collaborating on new papers are common uses of Facebook groups.
This is a summary of: Social media for fisheries science and management professionals: How to use it and why you should

Accessible at: https://marxiv.org/aey2j

Author:

David Shiffman

Added to MarXiv:

February 2018

Published: In press

Suggested Citation: How to use social media for fisheries science and management professionals. OCTO (2018). DOI: 10.17605/OSF.IO/3SKRE

See more MarXiv summaries at https://www.marxivinfo.org/ summaries

The MarXiv Team

Nick Wehner,

Director of Open Initiatives, OCTO

Raye Evrard,

Project Manager, OCTO

Allie Brown,

Project Associate, OCTO

Sarah Carr,

Chief Knowledge Broker, OCTO

John Davis,

President, OCTO

Correspondence

Nick Wehner

nick@octogroup.org

Financial support for MarXiv comes from the David and Lucile Packard Foundation. 


\section{MarXiv Summary}

MarXiv: The free research repository for the ocean \& marine-climate sciences

\section{Blogs}

Blogs are essentially online newspapers with a blog post representing an individual article. Blogs allow users to write about any kind of topic they like, directly to their readers. Wordpress is a common (free or paid) blogging platform. All you need is a name and description for your blog. Themes can be changed and customized to alter the look and feel of your blog.

Blogs are typically used by scientists to convey information the media often does not cover, to correct misinformation in the media, or to share new research results. Blog hosts often feature guest posts written by colleagues who lack their own blogs or wish to extend their influence.

\section{MarXiv}

Across all disciplines, papers available for free with a preprint (also known as "Green OA") are cited $30 \%$ more than the world average (DOI: $10.7287 /$ peerj. preprints.3119v1)

Share your research in MarXiv and the MarXiv Team may summarize it, just like this!

Step-by-step instructions on how to share your research in MarXiv are detailed at https://www.marxivinfo.org/ submission

Want to know when you can update your preprint with your postprint? Check our list of Self-Archiving Policies of Major Journals at https://www.marxivinfo.org/ policies

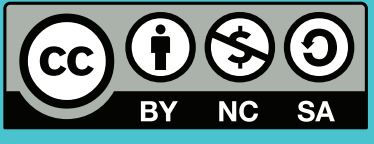

MarXiv is an ОСТO Initiative
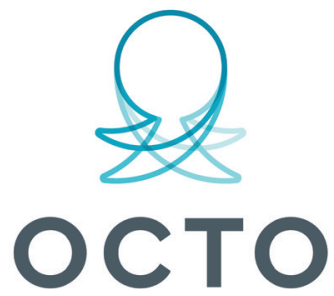

OPEN COMMUNICATIONS FOR THE OCEAN 